\title{
Risques et incertitudes sur le marché mondial des huiles de palme, palmiste et coprah
}

\section{Risks and uncertainties on world palm oil, palm kernel and copra markets}

Oléagineux, Corps Gras, Lipides. Volume 7, Numéro 2, 140-6, Mars - Avril 2000, Dossier : Afrique, plantation et développement

Auteur(s) : Tancrède VOITURIEZ

Résumé : Nous essayons dans cet article de fournir les grandes caractéristiques du marché mondial des huiles végétales, avec l'intention tout d'abord de décrire la contribution des oléagineux pérennes et son évolution dans la production et le commerce mondial des huiles, puis de comprendre la nature particulière des risques attachés à ces cultures. À partir de données historiques couvrant la presque totalité du siècle, nous verrons que la situation actuelle est une situation d'incertitude : la part des oléagineux pérennes dans l'échange stagne après avoir connu un cycle de vingt années de croissance, tandis que les deux risques fondamentaux que sont l'exposition aux fluctuations des prix mondiaux (on n'arrache pas ses arbres au gré des baisses de cours) et la rigidité de la productivité du travail (on ne sait pas mécaniser de manière satisfaisante la collecte des fruits) ne trouvent pas de solution satisfaisante. La première partie aborde ces points. Nous reviendrons dans un deuxième temps sur un exemple réussi de couverture du risque, dans le cas de l'huile de palme, avec l'exemple malaysien qui prouvera que des stratégies nationales de gestion de risque peuvent être efficaces, sinon indispensables, et complémentaires du développement d'initiatives privées. Qu'en est-il de l'Afrique et quelle est l'ampleur des risques et des contraintes qu'y rencontrent les cultures oléagineuses pérennes : ce sont les deux questions qui occuperont notre troisième et dernière partie, en réponse desquelles seront décrits la convention de Lomé et ses effets sur la production et le commerce oléagineux sur le continent africain. Nous conclurons sur l'opportunité pour l'Afrique d'augmenter ses parts de marché nationales et internationales à la lumière des éléments apportés dans les trois parties de cet article.

Summary : The supply of perennial oilseed crops, like palm, palm kernel and coconut, faces two major constraints that ordinary annual oilseed crops face in a much lower extent. The difficulty to reap off productivity gains because of the technical constraints attached to mechanising harvesting, firstly, and secondly, the exposure to world prices because of strong short term rigidity make altogether perennial crops production very risky. History proves however that supplying vegetable oils like coconut oil and more recently palm oil has encountered a paramount success in countries like Philippines, Malaysia or Indonesia. Focusing especially on the palm oil market, we try in this paper to describe and analyse the nature of the risk prevailing in perennial crops production as well as some successful strategies designed and implemented so far to mitigate such risk. Highlighting the role of the state in risk coping, the paper concludes on the possible lessons that could be learnt for Africa in the future with a state much weaker than it used to be in South East Asia and a new Lomé Partnership more in line with WTO rules.

Keywords : perennial crops, oilseeds market, vegetable oils market, historical data, risk, Lomé Convention. 


\section{ARTICLE}

\section{Grandes caractéristiques du marché mondial des huiles et graisses}

\section{Un secteur stratégique encouragé à l'origine par l'État}

La reconstitution de l'évolution sur près d'un siècle des échanges mondiaux d'huiles et oléagineux, mesurés en équivalent huile (oléagineux en équivalent huile + huile) à partir de différents annuaires ${ }^{1}$, montre distinctement la succession de trois " booms " qui, ces cinquante dernières années, ont propulsé, dans l'ordre, l'huile de soja à partir de la fin des années 50 , l'huile de palme à partir de la fin des années 60 et les huiles de colza et de tournesol à partir de la fin des années 70 sur un sentier de croissance beaucoup plus pentu que ce que le monde des huiles et graisses avait connu jusque-là. Le soja est aujourd'hui l'objet de $35 \%$ des échanges d'oléagineux et d'huile, contre $31 \%$ pour l'huile de palme et $20 \%$ pour le binôme colza et tournesol ${ }^{2}$. On peut rappeler que ces trois groupes d'oléagineux, leaders actuels du commerce des huiles végétales, sont des plantes à la production et à l'exportation desquelles on trouve, à l'origine, une incitation volontaire de l'État, incitation volontaire dont la manifestation s'observe encore sous la forme, par exemple, de prix plancher garanti (marketing loan américain), d'exemptions de taxes d'exportation ou encore d'exonérations fiscales (cas du secteur de la lipochimie en Malaisie). Ainsi des efforts publics de promotion et de développement du soja (États-Unis dès les années 30, Brésil et Argentine dans les années 80), du colza et du tournesol (Communauté européenne dans les années 60 et 70 ) et de l'huile de palme (Malaisie à partir des années 60, Indonésie à la fin des années 70). La figure 1 retrace l'accroissement exponentiel de l'échange de ces trois groupes d'huiles depuis 1906. On notera la stagnation des exportations de coco qui ne parvient pas à atteindre, dans la hiérarchie des huiles, son rang d'avantguerre, ainsi que le détaille la figure 2 .

\section{Deux cycles des cultures pérennes et tropicales dans les échanges mondiaux}

L'agrégation de nos données brutes permet par ailleurs de rendre compte de la part occupée par les oléagineux tropicaux et pérennes (palme, palmiste, coco) dans le commerce mondial sur le siècle écoulé. La figure 3 montre ainsi qu'après un cycle de croissance de 1909 à 1950, durant lequel la part de marché à l'exportation des oléagineux pérennes et tropicaux est passée de 27 à $60 \%$, un long cycle de déclin a réduit cette part à $25 \%$ en 1970. Ce cycle traduit tout d'abord les effets des politiques d'auto-suffisance américaines et le règne du soja déversé sur le marché mondial à titre commercial ou sous la forme d'aide alimentaire. II correspond également au délai de maturation du verger malaysien, alors en début d'expansion. Les années 90 terminent ce second cycle ; à $38 \%$, la part des cultures pérennes tropicales égale, sans le dépasser, son niveau de l'entre-deux-guerres car, entre temps, l'Europe s'est à son tour lancée dans la course à la production en développant colza et tournesol pour réduire son déficit commercial de corps gras. Cultures d'exportations, les cultures pérennes occupent une plus large part dans le commerce mondial que dans la production mondiale, comme l'indique la figure 4 où le cycle d'après guerre apparaît clairement mais culmine dans les années 90 à un niveau inférieur à celui observé dans le cas des échanges ( $27 \%$ contre $38 \%$ ). Un plateau, tant dans la production que dans l'échange, caractérise la situation des années 90 . Par quelle phase va s'amorcer le prochain cycle, phase ascendante ou descendante ? C'est une question à laquelle les dernières statistiques suggéreraient de répondre par la baisse, en raison notamment du 
tassement de la croissance des exportations indonésiennes d'huile de palme attendu après 2001. Ce tassement correspond aux palmiers " non plantés " après la destitution de Suharto en raison de l'incertitude politique et de la montée des taux d'intérêt à des niveaux records gelant tout investissement. L'atermoiement prolongé des investisseurs souligne le risque inhérent à la mise en culture d'oléagineux pérennes, de surcroît lorsque les meilleures terres ont été cultivées, par les fortes immobilisations en capital qu'elle suppose.

\section{Polarisation de la demande}

La demande en corps gras est extrêmement disparate dans le monde et fortement inégale, puisque les consommations d'huiles et graisses par tête et par an s'étirent de moins de $7 \mathrm{~kg}$ au Soudan et au Zimbabwe à plus de $40 \mathrm{~kg}$ dans I'Union européenne ou aux États-Unis. Des tendances communes semblent pourtant exister qui décrivent, dans chaque région, la transition alimentaire au cours du temps d'une consommation à base de blé et de féculents vers une consommation plus riche en viandes, en sucre et en corps gras [4]. L'accroissement du revenu et l'urbanisation, avec les changements de modes de consommation qu'ils entraînent, sont les déterminants les plus aisément observés et mesurés pour expliquer la transition alimentaire du " grain " au " gras ". L'extension brutale du marché que décrit la figure 1 est ainsi concomitante de l'accroissement brutal de la consommation d'huiles et graisses par tête en raison d'un changement de régime alimentaire survenu dans les années 30 et surtout dans l'immédiat après-guerre en Europe et dans les années 70 et 80 en Asie, notamment en Chine et en Inde. L'arrivée de nouvelles régions consommatrices et importatrices asiatiques se manifeste dès 1971 ; elle sera renforcée, dans le cas de l'huile de palme, par la politique malaysienne d'incitation aux exportations de produits raffinés, exonérés de taxe à partir de 1976 : repoussées par l'Europe qui protège ses raffineries par une taxe discriminatoire sur les importations d'huile transformée (triple de celle sur les huiles brutes), ces huiles prêtes à la friture sont achetées au Pakistan, en Inde, en Chine. Actuellement, près de $50 \%$ de I'huile de palme mondiale est consommée en Asie, contre moins de 12 \% en Europe. Les importations donnent des résultats comparables : les importations asiatiques représentaient moins de $20 \%$ des importations mondiales jusqu'en 1972-1973, pour fluctuer à partir de cette date entre 40 et $60 \%$. Les importations européennes représentaient plus de $60 \%$ avant 1972-1973. Elles se situent sous la barre des $20 \%$ depuis le début des années 80 . On notera pourtant qu'à côté de ce recul relatif, puisque exprimé en parts de marché, l'Union européenne reste le premier importateur d'oléagineux, le premier importateur d'huiles et graisses, et est le deuxième triturateur. Simplement, elle diversifie ses produits d'importations.

\section{Complémentarité des cultures pérennes et annuelles dans l'ajustement de l'offre à la demande}

Le marché des oléagineux possède la caractéristique unique de rassembler des cultures annuelles et des cultures pérennes dans un même espace d'échanges. Cette caractéristique lui confère des propriétés dynamiques particulières. À la tendance longue et prévisible de la production pérenne (dans la mesure où les statistiques existent et sont fiables, il n'est pas difficile dans un pays donné de prévoir les productions d'huile de palme par exemple à partir de données sur l'état du verger et sa superficie) s'ajustent les productions annuelles des deux hémisphères. La première est extrêmement peu réactive aux prix à court terme ; les secondes y sont beaucoup plus sensibles. Au total, et comme ce fut le cas depuis la suprématie de l'huile de palme dans l'échange ${ }^{3}$ à partir du milieu des années 80 , les cultures pérennes garantissent, indépendamment du prix, la fourniture d'un certain volume 
d'huile, qu'augmenteront, dans des proportions dépendant cette fois des prix, les cultures annuelles. Une sorte d'auto-régulation du marché, fondée sur la complémentarité des cultures annuelles et pérennes, permet ainsi à première vue d'éviter les longues pénuries - les prix montant, les oléagineux annuels sont rapidement plantés - ainsi que les longues périodes de surplus puisqu'à l'inverse, lorsque les prix baissent, les oléagineux annuels sont moins attractifs et leur surface se réduit. La réalité n'est pas tout à fait conforme cependant à ce schéma d'ajustement.

Ainsi, depuis deux ans, à côté de la croissance prévisible des exportations indonésiennes et malaysiennes, en raison notamment de l'arrivée à maturation de jeunes palmiers plantés à grande échelle en Indonésie à la toute fin de l'ère Suharto, le soja nord-américain particulièrement abondant maintient la pression sur les cours des huiles. L'équilibre que devrait posséder le marché avec, d'un côté, l'huile de palme fournie par une plante pérenne plantée et replantée dans une perspective de long terme et, de l'autre, les huiles tempérées telles que les huiles de soja, tournesol, colza, fournies par des cultures annuelles, est dans les faits extrêmement instable. Et l'ajustement de l'offre à la demande est imparfait. En baisse prononcée, les cours signalent la perspective de surplus. L'offre la plus élastique, l'offre de cultures annuelles, devrait se restreindre. Tout au contraire elle augmente, pour des raisons indépendantes du prix et directement liées au système des marketing loans qui assure aux fermiers américains, comparativement à d'autres produits tels que le blé ou le maïs, la moins mauvaise rétribution pour la production d'oléagineux. Le risque fondamentalement lié aux prix et à la volatilité des cours n'est donc pas atténué sur le marché des huiles en dépit de caractéristiques singulières qui l'eussent permis.

Comment, dès lors, le risque que représentent, d'une part, l'immobilisation durant plusieurs années d'un investissement lourd sans gain de productivité du travail évident et, d'autre part, de fortes variations de recettes induites par les fluctuations de cours mondiaux peut-il être surmonté ? La perspective d'une croissance soutenue de la demande par l'effet mécanique d'une hausse du revenu par tête et de l'accroissement démographique devrait encourager les investissements dans le secteur oléagineux. Comme nous l'avons signalé, le plateau atteint par les oléagineux pérennes depuis bientôt dix ans indique grossièrement que le risque perçu dans ce secteur est plus important que dans le secteur des oléagineux annuels. Ce risque, par une intervention publique ciblée, peut pourtant être réduit. La stratégie victorieuse de couverture du risque appliquée en Malaisie depuis trente ans nous fournit un exemple remarquable de partage des responsabilités, entre secteur public et secteur privé, dans la prise en charge du " risque pérenne " que nous avons mentionné. Nous verrons ensuite s'il est duplicable dans d'autres régions.

\section{Couverture du risque : l'exemple malaysien}

Aux prises avec une culture d'exportation pérenne, exposée à la contrainte extérieure forte que sont les variations des prix mondiaux et à une contrainte intérieure tout aussi prégnante qui est la productivité du travail (on ne sait pas mécaniser de manière satisfaisante la collecte des fruits), la stratégie de l'État malaysien fournit un exemple inégalé de conquête de marchés extérieurs et de couverture du risque prix. La Malaisie, rappelons-le, est le premier exportateur mondial d'huile de palme, avec actuellement $65 \%$ des parts du marché mondial de cette huile. La stratégie est constituée de trois points : 
- privilégier les activités intenses en capital, qui permettent d'augmenter les gains de productivité (ce sont les activités de transformation) ;

- favoriser la diversification des exportations qui doivent couvrir plusieurs secteurs d'activités et, au sein d'un même secteur, plusieurs produits ;

- créer un marché à terme de l'huile de palme brute (Kuala Lumpur Commodity Exchange ou KLCE) pour offrir aux producteurs, transformateurs, exportateurs locaux un outil de gestion du risque prix et un climat favorable aux investissements.

C'est ainsi que, après l'exportation d'huile de palme brute à partir des plantations de l'État fédéral (plantations du Felda) venues renforcer le secteur privé établi depuis 1918, l'industrie de première transformation comme la raffinerie est encouragée au début des années 70 par des investissements directs et la réduction de la taxe à l'exportation. Dans une troisième phase plus récente, l'industrie de transformation avancée comme la lipochimie ${ }^{4}$, à haute valeur ajoutée, est favorisée par l'État fédéral à travers des joint-ventures, un statut fiscal préférentiel dit de " pionnier » et des taxes réduites ou supprimées à l'exportation. Investissement direct en plantation, investissement conjoint avec le secteur privé dans les raffineries, incitation fiscale enfin pour le développement de la lipochimie grâce à des capitaux privés et étrangers et l'importation de main-d'œuvre qualifiée, la stratégie de l'État fédéral malaysien, inscrite sur plus de vingt ans, a permis à la péninsule d'imposer la culture du palmier comme culture de gestion de risque en soi, en l'ajoutant à l'hévéa et à la production d'étain. Elle a suscité ensuite, au sein du secteur, la réduction des risques par la diversification et la multiplication des produits d'exportation dérivés de l'huile de palme (huile raffinée, fractions liquides et solides, acides gras, tensio-actifs) ainsi que par la création, au tout début des années 80 , d'un marché à terme de l'huile de palme brute à Kuala Lumpur (figure 5).

L'Indonésie connaît une évolution similaire et quelque peu décalée. Plus proche de la situation africaine, si l'on considère l'importance de sa demande intérieure d'huile de palme (environ $50 \%$ des volumes produits sont consommés sur place), elle a développé les investissements productifs en plantation et protégé son industrie de transformation par des achats à prix garantis. La similitude s'arrête là cependant, l'Indonésie n'ayant jamais souhaité développer un secteur à très haute valeur ajoutée par crainte de voir disparaître vers l'exportation une part trop importante de sa production qu'elle affecte en priorité à la consommation domestique. Concluons en transition avec le paragraphe suivant en précisant que le succès de la stratégie en trois étapes que l'on vient de décrire aurait été entamé dans d'autres conditions de sols et de climat, conditions "idéales " dans la péninsule malaysienne et à Sumatra.

\section{Risques et contraintes en Afrique}

Dans des conditions de sols et de climat un peu moins favorables ${ }^{5}$, quels sont les risques particuliers à l'Afrique dans l'investissement en palmeraies ? Comment les couvrir ? On peut pour répondre à ces questions s'en poser une autre, et se demander par exemple dans quelle mesure la stratégie malaysienne est reproductible. Mais connaissant les marges de manœuvre laissées par les programmes d'ajustement structurel aux gouvernements africains pour la perception et la dépense des recettes publiques, une réponse froide et lapidaire consisterait à énumérer les faiblesses du continent : absence de marché à terme, désengagement de l'État, défaillances des infrastructures de transport et désincitation, en conséquence, à l'installation d'usines de transformation. Le secteur 
privé doit seul prendre en charge les risques sans que tous soient échangeables sur un marché - ainsi du risque prix, qui oblige à des contrats moins transparents de gré à gré -, d'où la relative frilosité des investisseurs privés et la stagnation des surfaces plantées de cultures pérennes. Une autre réponse consisterait à ce que l'Afrique se donne d'autres objectifs que les marchés extérieurs et, partant, qu'elle développe des stratégies à la couverture du risque autres que celles mises en place par la Malaisie dans la mesure où celle-ci visait à la conquête, précisément, de marchés extérieurs. Le délabrement des infrastructures de transport semble être un élément essentiel des deux stratégies : il agit en effet comme une barrière à l'échange. En empêchant les produits importés d'arriver à moindre frais dans les villes enclavées et loin de la mer, il favorise les planteurs locaux s'il s'en trouve. À l'inverse, en renchérissant le prix à l'exportation d'huiles acheminées depuis l'intérieur, il pénalise les producteurs locaux et les exportations extra-continentales. Miser toutefois sur les seules défaillances des infrastructures de transport pour justifier la seconde stratégie, stratégie visant à la conquête du marché continental, est un peu maigre et relèverait presque d'un cynique désabusement. Nous énumérerons les points qui pourraient militer pour l'une ou l'autre stratégie, mais il est évident qu'elles ne sont aucunement exclusives l'une de l'autre.

\section{L'Afrique en retrait}

Quelques indicateurs tout d'abord illustrent le retrait du continent africain dans le commerce mondial d'huiles et graisses. Nous privilégierons l'huile de palme, par son importance sur le marché mondial et, historiquement, son origine africaine. De moins de $1 \%$ au début des années 60 , les exportations malaysiennes et indonésiennes d'huile de palme représentent actuellement environ 80 $\%$ des exportations mondiales. La part des exportations africaines était de $99 \%$ au début des années 60. Elle est maintenant inférieure à $10 \%$ (figure 6).

Les rendements d'huile de palme ont par ailleurs atteint en 1999, en moyenne mondiale, 3,33 tonnes d'huile de palme par hectare. Les performances du verger malaysien se sont accrues en 1999 de près de $20 \%$, à 3,88 tonnes par hectare, du jamais vu depuis les exceptionnelles 4,05 tonnes par hectare constatées en 1982 après l'introduction du " weevil " pollinisateur dans les plantations de la péninsule (la réaction des arbres l'année suivante fut brutale, en baisse de $20 \%$ ). On attend un rendement moyen en Malaisie en 2000 situé aux alentours de 3,6 tonnes par hectare, en baisse de 6,4 \% mais encore au-dessus de la moyenne des cinq années 1993-1997. Les rendements indonésiens se situaient en 1999 à 3,2 tonnes par hectare en 1999. Les rendements sont estimés aux alentours de 2 tonnes par hectare en moyenne en Côte d'Ivoire et au Nigeria.

Enfin, avec un prix de la main-d'œuvre très compétitif en Indonésie et en Malaisie, l'Afrique ne peut espérer compenser ses médiocres rendements en moyenne par un moindre coût de ses facteurs et se mesurer aux pays de l'Asie du Sud-Est sur le marché mondial, même si ce qui est vrai en moyenne n'est pas toujours vrai dans le détail.

\section{Absence de marché de couverture du risque prix}

Aucun marché à terme d'huiles végétales n'existe en Afrique et les contrats sont, pour cette raison, des contrats de gré à gré. Le risque de contrepartie que comportent de tels contrats (aucune chambre de compensation ne vous assure de la fiabilité de votre fournisseur) repose essentiellement sur le négociant. Or des stratégies de réduction du risque de contrepartie par le négociant, d'un côté, et le développement de plantations dites "villageoises " couvrant un faible nombre d'hectares, de 
l'autre, peuvent être contradictoires et ne pas déboucher sur la signature de contrats, les volumes et la stabilité dans la qualité des produits requis pour l'exportation ne se trouvant pas de manière certaine parmi ces dernières.

\section{Villes côtières et orientales approvisionnées par l'Asie}

Les statistiques sur la pénétration des huiles de palme asiatique dans le marché africain pour l'année 1999 montrent qu'un nombre important d'États côtiers, ainsi que les pays de l'Afrique orientale et du Maghreb, préfèrent l'approvisionnement régulier et sûr que leur fournissent la Malaisie et I'Indonésie à celui proposé en Afrique (figure 7). Cette situation se retrouve pour de nombreux autres produits : riz, oignon ou céréales. Des pays comme le Kenya ou l'Afrique du Sud sont ainsi devenus en une décennie des importateurs d'huile de palme significatifs à l'échelle mondiale. Des prix attractifs par rapport aux huiles de soja ou de tournesol, une offre diversifiée (huile brute ou raffinée), des produits de qualité, une agressivité commerciale permanente des exportateurs asiatiques sont quelques-unes des raisons pouvant expliquer l'ampleur et la rapidité de cette évolution. Un surcoût des huiles africaines acheminées jusqu'à destination sur les huiles asiatiques transportées au départ de Port Klang, Singapour ou Medan sur les lignes maritimes extrêmement compétitives est également une explication déterminante de l'engouement que manifestent un grand nombre de pays africains pour les huiles asiatiques. II ne faudrait cependant pas négliger les irréversibilités des modes de consommation urbain qui, en privilégiant une huile de friture raffinée, inodore et incolore, comme c'est le cas au Maghreb et en Afrique orientale et australe, laissent peu de chance à l'huile de palme brute, l'huile rouge, africaine, d'entrer dans la constitution de leurs plats.

\section{Convention de Lomé et fin annoncée des préférences commerciales}

En ouvrant le marché européen, sans aucune perception de droits de douane, aux produits non transformés d'origine des pays de l'Afrique, des Caraïbes et du Pacifique (ACP) (tableau 1), ainsi qu'en fournissant des outils financiers de gestion de l'instabilité des recettes d'exportation (Stabex), la convention de Lomé est en soi un dispositif anti-risque. Les négociations entre l'Union européenne et les pays ACP se sont officiellement achevées le 3 février 2000, peu de jours avant l'échéance de la quatrième convention de Lomé qui survenait le 29 février 2000 et l'entrée en vigueur d'un nouvel accord. Il est prévu que la nouvelle convention - ou accord de partenariat, la dénomination définitive n'étant pas encore fixée - soit signée le 31 mai aux Fidji par les quinze pays de l'Union européenne et leurs 71 partenaires ACP. Les pays ACP ont obtenu que soit prorogé pendant une période préparatoire de huit ans le régime de préférences commerciales non réciproques dont ils ont bénéficié jusqu'ici. Cette "victoire " des pays ACP ressemble à un délai de grâce consentie par I'Union européenne tant on sait que la disparition du régime de préférences, qui est la clé de voûte des accords de Lomé, est à terme acquise.

À la période préparatoire de huit ans durant laquelle les préférences commerciales non réciproques resteront en vigueur, succéderont en effet douze années de transition pendant lesquelles seront progressivement appliqués des accords de partenariat économique (APER) entre l'Europe et les pays ACP regroupés en blocs régionaux.

Grâce au nouvel accord commercial, I'Union européenne souhaite officiellement promouvoir l'intégration régionale, favoriser l'investissement et améliorer la compétitivité des économies ACP. Elle conserve avec celles-ci une relation économique et commerciale privilégiée en évitant, et c'est la 
nouveauté, de se mettre en position délicate vis-à-vis de I'OMC. II n'a échappé à personne pour autant que, en raison de la franchise de droits de douane sur le marché européen de la quasi-totalité des exportations ACP, l'enjeu principal du nouvel accord commercial réside, pour les pays ACP, dans l'ouverture de leurs marchés aux importations européennes. Or les contraintes internes de production et le faible développement de marchés tels que les marchés des services limitent fortement les capacités de réaction des économies ACP à la concurrence européenne. L'accord comporte également des risques pour les exportateurs de produits tropicaux. Les pays ACP non PMA qui ne signeront pas l'accord de libre-échange avec l'Union européenne tomberont dans le système de préférence généralisé (SPG). Ce régime d'accès au marché européen, géré unilatéralement par I'Union européenne, est pour le moment moins favorable que le régime de Lomé. II pourrait entraîner des pertes sérieuses de préférences, donc de compétitivité, pour certains secteurs d'activité. Des études ont montré en effet que les pays qui tirent le plus avantage des préférences sont parmi les pays ACP ceux qui sont parvenus le plus à diversifier leurs exportations. La disparition des préférences est alors d'autant plus douloureuse qu'elle incite à l'extraversion et à la spécialisation des agricultures ACP dans les filières " traditionnelles " d'exportation (café, cacao, coton, huile de palme) que la conjoncture rend spectaculairement peu rentables et très risquées. L'Europe n'a au total que le libre-échange à offrir à ses partenaires du Sud, ce qui, pour un programme d'action politique et de développement, ressemble plus à l'absence d'idée et à l'indifférence qu'à une quelconque volonté politique de construire un partenariat économique viable, autre chose que la valorisation d'avantages comparatifs éculés - le libre-échange, ou la solution par défaut que l'on trouve dans tous les manuels.

\section{L'introversion de l'Afrique déjà en chemin ? Quelques enseignements de Lomé}

L'Afrique, sans accès prioritaire au marché européen, doit-elle se tourner vers elle-même ? Le marché continental africain est-il donc le marché le plus prometteur des futures plantations ? Les données d'échanges entre I'UE et les pays ACP rassemblées par Eurostat apportent quelques précisions intéressantes sur les deux stratégies soulevées en introduction de cette section. Un détail en est donné par Ribier et Voituriez dans ce même numéro.

L'évolution sur dix ans des échanges UE-ACP confirme, en outre, que les parts de marchés de l'huile de palme et des huiles lauriques ACP diminuent dans I'Union européenne. Celle-ci s'approvisionne de moins en moins parmi les ACP, au profit des Philippines et de l'Asie du Sud-Est.

On doit souligner par ailleurs la présence faible ou nulle, sur les marchés hors ACP et hors Union européenne, des produits oléagineux des ACP. La virulence des attaques de I'American Soybean Association (ASA) à l'encontre des huiles tropicales dans les années 70 et 80 explique encore la faible part de marché qu'occupe l'huile de palme, d'où qu'elle provienne, sur le marché nord-américain, tandis que la vigueur du secteur oléagineux (soja, tournesol) au Brésil et en Argentine réduit les parts de marché des produits importés en Amérique du Sud. Les importations d'huile de palme représentent ainsi environ $10 \%$ des importations d'huile végétale des États-Unis tandis que les importations américaines d'huile de palme représentent à peine $2 \%$ des importations mondiales de ce produit. La totalité des importations d'huile de palme et des huiles lauriques aux États-Unis provient d'Asie du Sud-Est et des Philippines. La situation est identique en Amérique du Sud qui dispose de surcroît de quelques fournisseurs de ces produits à proximité, comme la Colombie et l'Équateur. 
Notes

${ }^{1}$ Les annuaires de I'Institut international d'agriculture (IIA, ancienne FAO) [1], divers numéros du Bulletin des Matières Grasses [2] et de la revue Oléagineux [3], consultés dans les bibliothèques du Cirad à Nogent et à Montpellier, ont permis de créer des séries annuelles par grands produits jusqu'en 1959, date à laquelle Oil World entamait sa parution et complétait les séries.

${ }^{2}$ Si l'on considère uniquement les huiles, et non plus les huiles et les oléagineux convertis en équivalents huiles échangés dans le monde, la hiérarchie est quelque peu modifiée, l'huile de palme se plaçant en tête ( $43 \%$ des échanges mondiaux d'huiles végétales) devant l'huile de soja (24\%), I'huile de tournesol (11 \%); suivent coco et colza (6,5\%), enfin arachide (1\%) et coton, lin, ricin, sésame $(<1 \%)$.

${ }^{3}$ Échanges d'huiles végétales uniquement.

\section{CONCLUSION}

La situation, en conclusion, peut paraître bien sombre pour l'Afrique en raison, d'une part, de la prévalence des risques particuliers que comporte la mise en culture d'oléagineux pérennes, et que nous avons mentionnés, et, d'autre part, parce que, en dépit d'accords commerciaux préférentiels, I'Afrique ne parvient pas à conserver en moyenne ses parts de marché extérieur. L'introversion de I'Afrique n'est pourtant pas une solution, tout comme le " tout Europe » que favorisait Lomé n'en n'était pas une, ainsi que les statistiques d'échange l'ont montré. La valorisation des huiles tropicales en Europe, pour des motifs techniques, comme dans le cas des huiles de palme ou des huiles lauriques qui ne connaissent dans de nombreux usages industriels de concurrentes tempérées ou, pour des raisons commerciales, par un accès privilégié au marché européen, reste en effet une opportunité réelle pour les pays d'Afrique, à condition que des efforts (sur la qualité, les infrastructures, la stabilité des rendements) soient parallèlement poursuivis par ces derniers et avec l'aide des bailleurs de fonds pour accroître les parts de marché à l'intérieur du continent au sein duquel, et l'argument est irrésistible, la croissance de la demande devrait à terme dépasser celle observée en Europe où le taux de consommation de lipides par tête se rapproche d'une asymptote (à $50 \mathrm{~kg}$ par tête, contre environ $10 \mathrm{~kg}$ en Afrique).

\section{REFERENCES}

1. IIA (1939). La production et le commerce international des huiles et graisses. Série des monographies sur les principaux produits agricoles du marché mondial, $n^{\circ} 5$ : Rome : IIA.

IIA, Annuaire international de statistique agricole, diverses années de 1909 à 1939, Rome : IIA.

2. Bulletin des Matières Grasses (1917-1946). Marseille : Institut Colonial.

3. Oléagineux (1947-1994). Paris.

4. VOITURIEZ T (1999). L'huile de palme et son marché : la modélisation de la volatilité. Thèse de doctorat en Sciences économiques, Université Paris I Panthéon Sorbonne.

5. AFD-CIRAD (1995). L'huile de palme dans les pays du golfe de Guinée et les besoins en replantation 
à long terme. Montpellier : CIRAD, 2 vol ; $210 \mathrm{p}$.

6. HARTLEY CSW (1967). The Oil Palm, Tropical Series. Longman Group Limited.

Oil World (1958-2000). Editions Mielke, Hambourg.

\section{Illustrations}

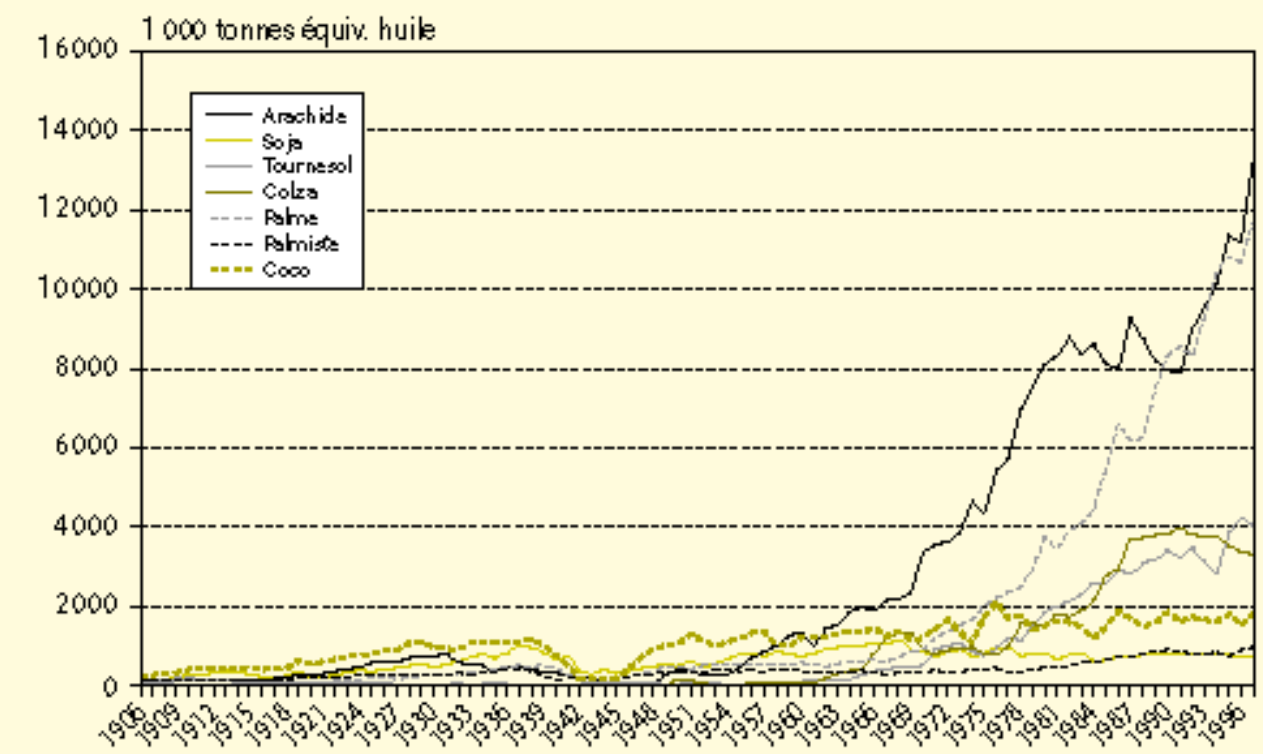

Figure 1. Commerce mondial des huiles et oléagineux, 1906-1996. 


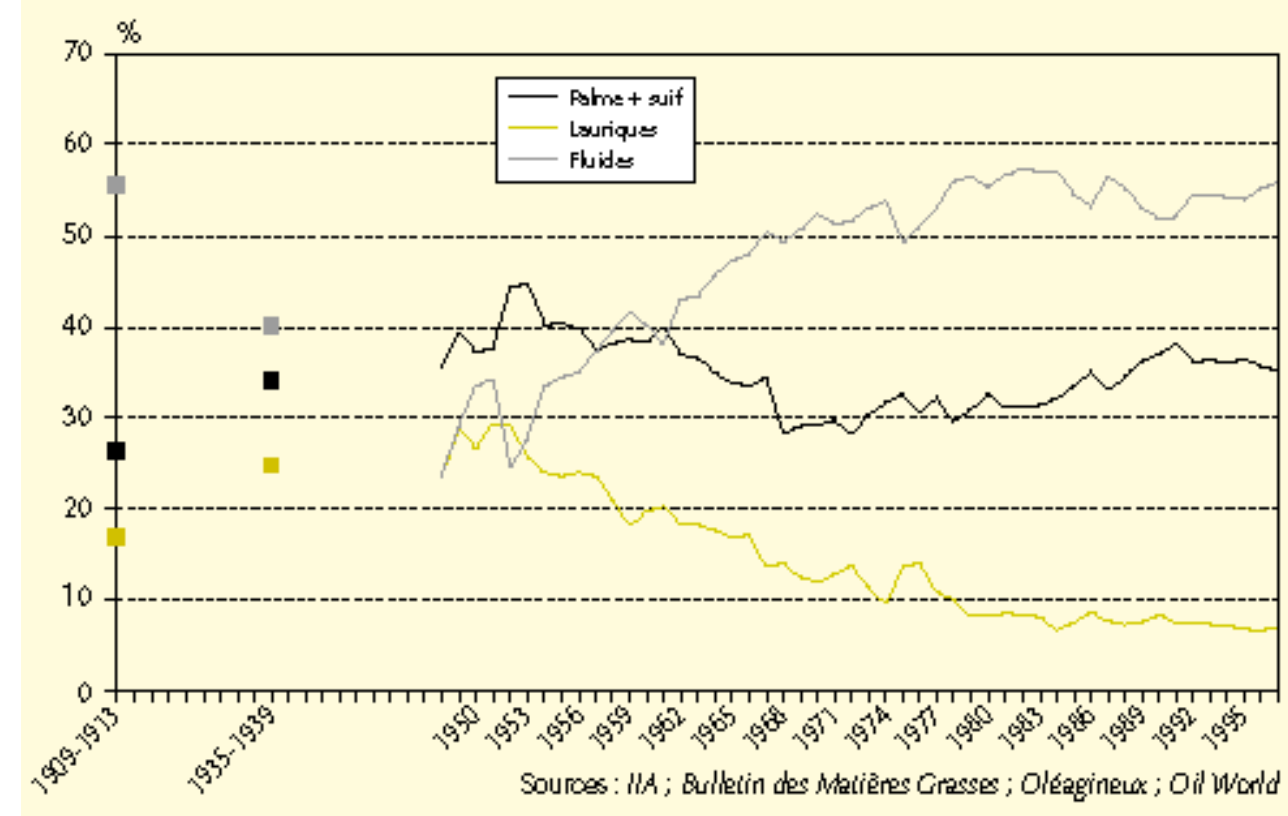

Figure 2. Distribution des trois groupes d'huiles et graisses dans le commerce mondial d'huiles et oléagineux (en équivalent huile).

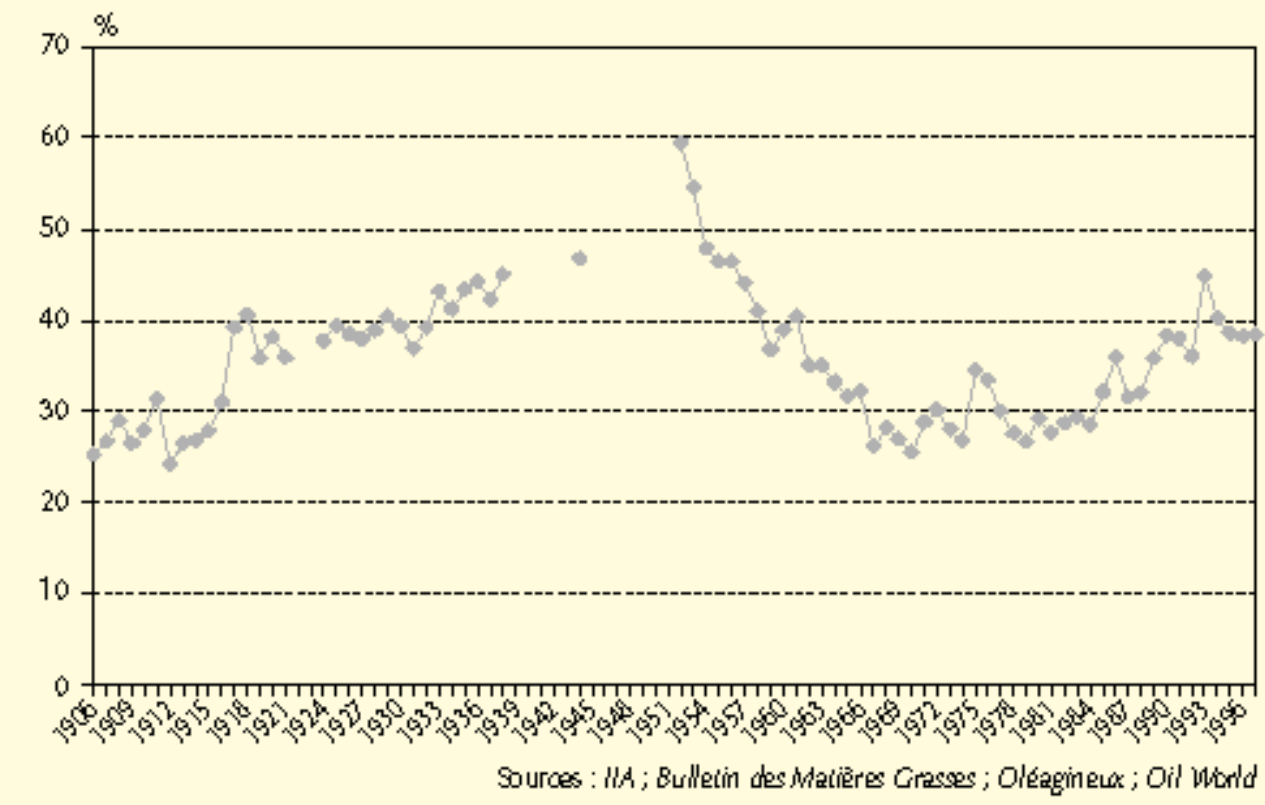

Figure 3. Part des oléagineux tropicaux pérennes (palme, palmiste, coprah) dans le commerce mondial des huiles et oléagineux (équiv. huile). 


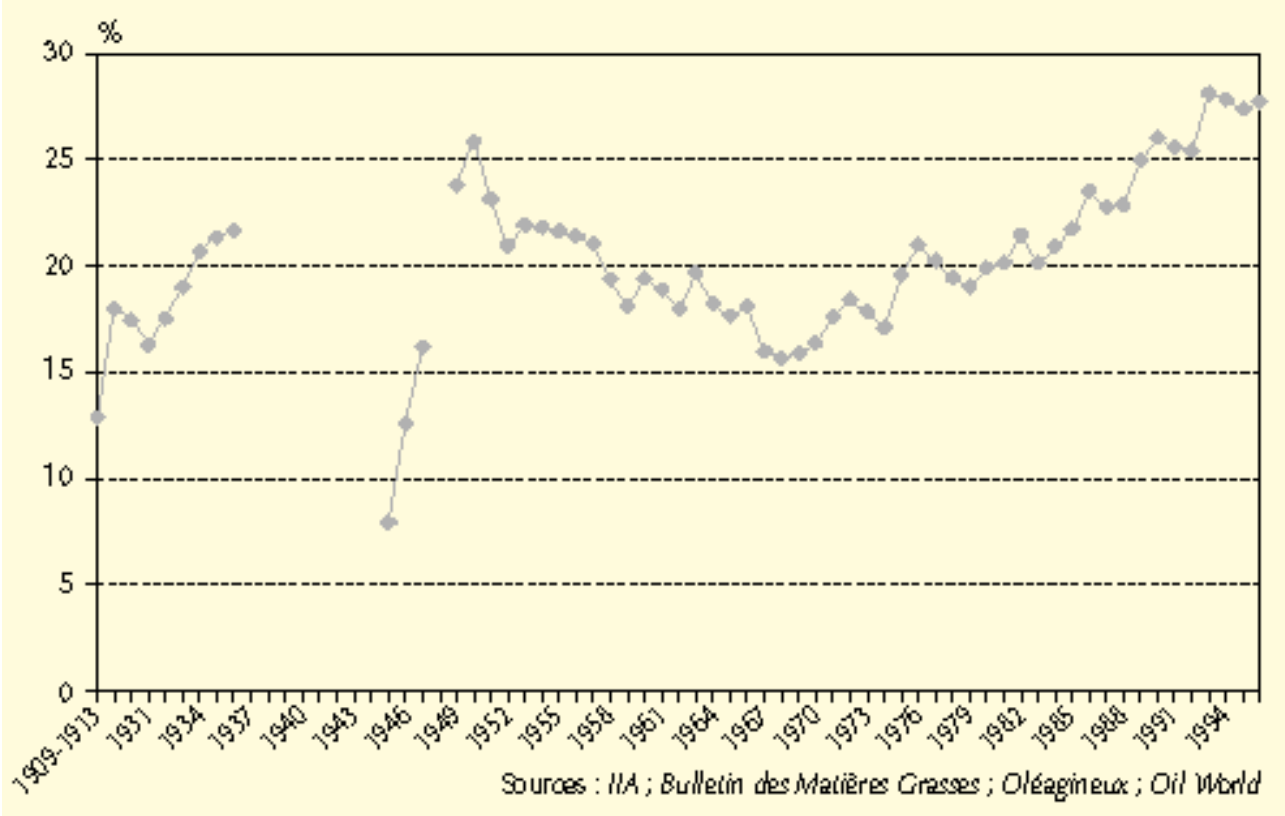

Figure 4. Part des oléagineux tropicaux pérennes (palme, palmiste, coprah) dans la production mondiale d'huiles végétales.

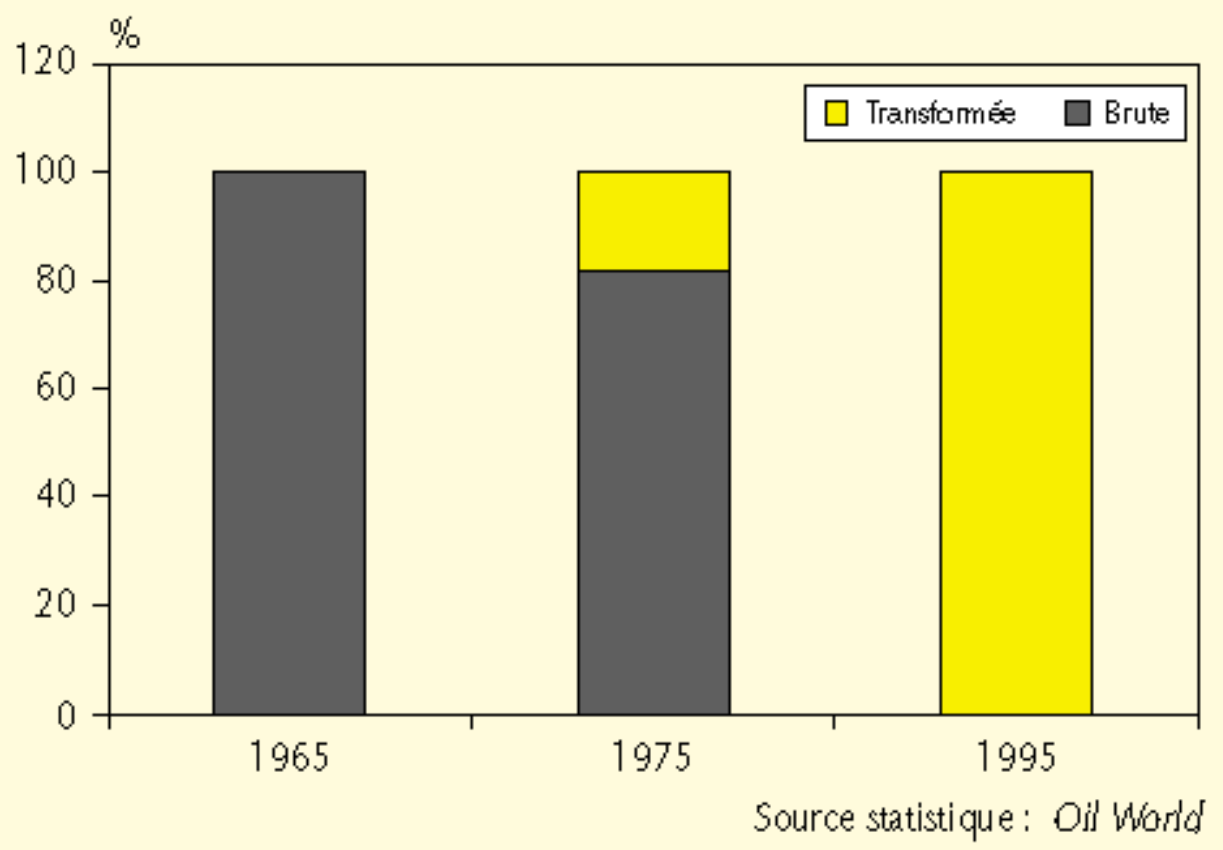

Figure 5. Exportations malaysiennes d'huile de palme brute et d'huile de palme 


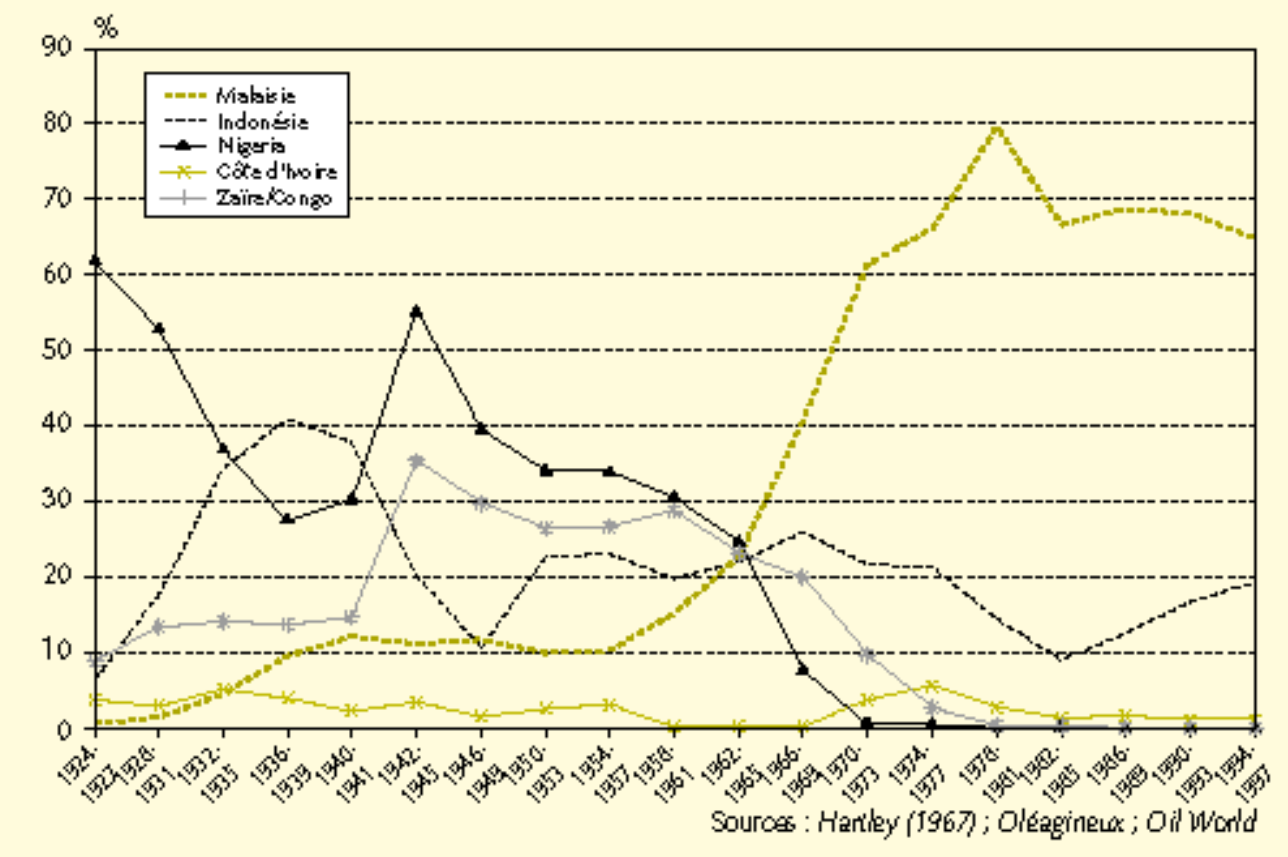

Figure 6. Parts de marché à l'exportation d'huile de palme, 1924-1997. 


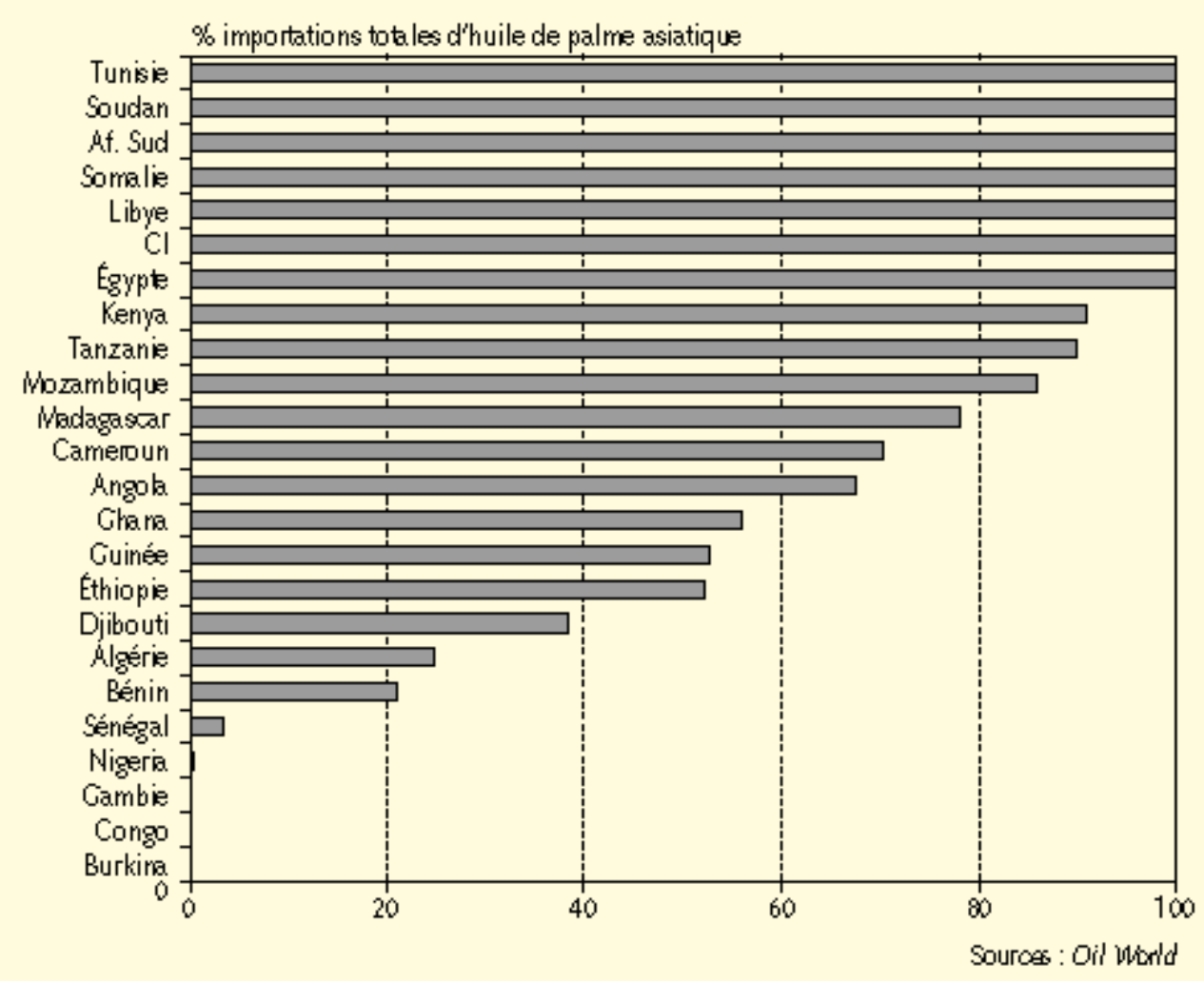

Figure 7. Importations africaines d'huile de palme asiatique (1999).

\begin{tabular}{|lcccc}
\hline Huile & Brute NA & Brute alimentaire & Autre NA & Autre alimentaire \\
\hline Soja & 3,2 & 6,4 & 5,1 & 9,6 \\
Arachide & Exemption & 6,4 & 5,1 & 9,6 \\
Palme & Exemption & 3,8 & 5,1 & 9 \\
Toumesol & 3,2 & 6,4 & 5,1 & 9,6 \\
Coton & 3,2 & 6,4 & 5,1 & 9,6 \\
Coco & 2,5 & 6,4 & 5,1 & 9,6 \\
Palmide & 3,2 & 6,4 & 5,1 & 9,6 \\
\hline
\end{tabular}

Tableau 1. Taux des droits conventionnels à l'importation (\%) dans l'Union européenne au 31-12-99

Source : Journal Officiel des Communautés européennes L 278, 28 octobre 1999. NA = non alimentaire. 